\title{
La FMH soutient l'initiative «Protection contre le tabagisme passif»
}

\section{Christine Romann}

Membre du Comité central de la FMH, Responsable du domaine Prévention et promotion de la santé
Lors du lancement, le 25 mai 2009, de l'initiative pour la «Protection contre le tabagisme passif», la loi fédérale correspondante avait déjà été approuvée, mais son ordonnance se trouvait encore en consultation et sa teneur n'était toujours pas connue. La loi veut protéger la population contre les effets néfastes du tabagisme passif et prévoit des interdictions de fumer dans tous les espaces fermés accessibles au public et ceux servant a rejeté par $55 \%$ des voix l'initiative populaire «Protection contre le tabagisme passif» pour approuver une contre-proposition du Grand Conseil prévoyant des locaux et des établissement fumeurs, alors que l'initiative n'autorisait que les locaux fumeurs. Doiton en conclure que la protection contre la fumée passive est une sorte de révolution culturelle qui ne s'implantera pas du jour au lendemain? Il n'y a pas si

\section{Les délégués ont estimé que la protection contre le tabagisme passif faisait partie des préoccupations principales du corps médical}

de lieu de travail à plusieurs personnes, tout en accordant aux cantons la possibilité d'édicter des dispositions plus strictes en la matière. L'éventualité d'une réglementation fédérale semblait donc reléguée aux calendes grecques. Au moment de la publication de l'ordonnance, fin octobre, les cercles intéressés durent constater que la protection des travailleurs était passée à la trappe (le lobby du tabac est-il en cause?). En effet, alors que la loi stipulait que les travailleurs ne pouvaient travailler qu'exceptionnellement dans les locaux fumeurs, l'ordonnance autorisait les établissements fumeurs et le service dans les locaux fumeurs. La réponse à la procédure de consultation formulée par la FMH avait critiqué cette proposition; mais en vain.

Dans les cantons, les débats font encore et toujours rage. Les points litigieux concernent presque toujours les espaces fermés accessibles au public, les lieux de travail et la restauration. C'est principalement le maintien d'établissements et de locaux fumeurs qui est contesté. Doivent-ils exister? Et dans le cas des locaux fumeurs, le service doit-il y être assuré ou non? Et quel est le contexte cantonal à la fin de l'année 2009? 18 cantons disposent de leur propre législation en la matière dont 15 (AR, BE, BL, BS, FR, GE, GR, NE, SG, SO, TI, UR, VD, VS, ZH), qui représentent en tout les trois quart de la population suisse, ont édicté des dispositions plus strictes que la loi fédérale. Certaines de ces réglementations ont été votées par le parlement, d'autres ont été légitimées par vote populaire.

Seul le Jura fait exception à la règle. En septembre 2009 , son parlement a rejeté une motion demandant la création d'une loi pour la protection contre le tabagisme passif.

Le résultat des votations porte à l'occasion sur des détails. Ainsi, le 17 mai 2009, le canton de Thurgovie longtemps en effet, la publicité pour les cigarettes faisait rimer consommation de tabac et soif masculine de liberté tout en alimentant le mythe (à grand renfort d'images de paysages à couper le souffle) selon lequel une restriction de la consommation de tabac équivaudrait à une privation de liberté.

Pour l'heure, six cantons seulement prennent la protection des travailleurs suffisamment à cœur pour n'autoriser que la création de locaux fumeurs sans service.

Par sa proposition, à fin 2008, de lancer une initiative populaire, la Ligue pulmonaire suisse a suscité des avis mitigés dans les cercles intéressés. Ces divergences d'opinion ne portaient pas sur le contenu - incontesté pour la plupart - mais sur la stratégie à adopter. La question qui se posait à l'époque était de savoir s'il ne valait pas mieux attendre la publication de l'ordonnance, et s'il n'était plus judicieux de laisser agir les cantons, dans le but d'économiser des forces et du temps. Le Comité central de la FMH partageait cet avis en décembre 2008, en prévision aussi des débats au sujet de la Loi sur la prévention, qui s'annonçaient épuisants.

L'initiative est désormais lancée et les efforts en vue de trouver une autre solution pour un avenir sans fumée se sont évanouis. En six mois, les auteurs de l'initiative ont récolté plus de 85000 signatures et espèrent qu'elle aboutira en mars 2010. Au vu de cette nouvelle réalité et suite à une longue discussion, l'Assemblée des délégués de la FMH s'est déclarée favorable à l'initiative. Les délégués ont estimé que la protection contre le tabagisme passif faisait partie des préoccupations principales du corps médical et ont approuvé l'initiative à une large majorité. 